\title{
Fundamentals of Base Station Availability in Cellular Networks with Energy Harvesting
}

\author{
Harpreet S. Dhillon, Ying Li, Pavan Nuggehalli, Zhouyue Pi, and Jeffrey G. Andrews
}

\begin{abstract}
We develop a new tractable model for $K$-tier cellular networks, where each base station (BS) is solely powered by a self-contained energy harvesting module instead of a conventional power-line source. The BSs across tiers differ in terms of the energy harvesting rate, energy storage capacity, transmit power and deployment density. Since a BS may not always have enough energy, it may need to be kept OFF and allowed to recharge while its load is served by the neighboring BSs that are ON. Using tools from random walk theory and stochastic geometry, we characterize the fraction of time each type of BS can be kept ON, termed availability, for general uncoordinated strategies, where each BS toggles its ON/OFF state independently of the others. As a part of our analysis, we model the temporal dynamics of the energy level at each BS as a birth-death process, derive energy utilization rate for each BS class, and use hitting/stopping time analysis to study availabilities. We prove that there is a fundamental limit on the availabilities, which cannot be surpassed by any uncoordinated strategy. As a part of the proof, we construct the strategy that achieves this limit.
\end{abstract}

\section{INTRODUCTION}

Several parallel trends have made the possibility of a selfpowered BS realistic in the near future. First, BSs are rapidly miniaturizing, and being deployed ever-more densely and opportunistically [1]. This means they require significantly less transmit power. Second, due to the increasingly bursty nature of user traffic, the loads on BSs will experience massive variation in time and space [2], which means that many BSs can, in principle, be turned OFF most of the time and only be requested to wake up intermittently based upon the traffic demand. Third, energy harvesting techniques, such as solar power, are rapidly becoming cost-effective, due both to technological improvements as well as market forces, such as increasing costs and taxes on conventional power sources, and subsidies and regulatory pressure for greener techniques. Fourth, high-speed wireless backhaul is rapidly becoming a reality for small cells [3]. Therefore, being able to avoid the constraint of requiring a wired power connection is even more attractive, since it would open up entire new categories of lowcost "drop and play" deployments, especially of small cells.

Although there has been significant advancement in the study of sensor and mobile ad hoc networks with self-powered transmitters, see, e.g., [4], [5], our understanding of the cellular networks in a similar setting is severely limited. This is partly due to the fact that conventional cellular networks

H. S. Dhillon and J. G. Andrews are with the Wireless Networking and Communications Group (WNCG), The University of Texas at Austin, TX, USA (email: dhillon@utexas.edu and jandrews@ece.utexas.edu). Y. Li and Z. Pi are with Samsung Research America, Richardson, TX (email: \{yli2, zpi\}@sta.samsung.com). P. Nuggehalli is with the Mobile and Wireless Group of Broadcom Corporation (email: pavann@broadcom.com). comprised exclusively of big macro BSs that required fairly high power and it made little sense to study them in the context of energy harvesting. Nevertheless, there has been some recent advancement in the understanding of a pointto-point communication link with self-powered transmitter, e.g., [6] studies the optimal rate adaptation to minimize total packet delivery time by modeling both the packets and the energy arrivals at the transmitter as random processes, [7] additionally incorporates the effect of finite energy storage capacity, and [8] further incorporates the effect of wireless fading channels. Clearly, the current cellular networks, with heterogeneous infrastructure and unique characteristics such as scheduling and cell selection, cannot be studied in the simple setups discussed above. This motivates the need for a fundamentally new framework and associated analytical tools to study self-powered cellular networks, which is the main focus of this paper.

We consider a general self-powered heterogeneous cellular network $(\mathrm{HCN})$, where several types of small cells may coexist with macrocells. Starting with the $K$-tier HCN model proposed in [9], where BSs across tiers differ in terms of the transmit power and deployment density, we additionally incorporate differences in the energy harvesting rate and energy storage capacity. For example, in a future two tier $\mathrm{HCN}$, macrocells may have a more aggressive energy harvesting module and a higher energy capacity than small cells. Modeling the locations of each class of BSs by an independent Poisson Point Process (PPP), we use tools from stochastic geometry to derive the energy utilization rate for each BS class. Under the assumption that the BS operational policies are decided over a much longer time scale than the one on which cell selection decisions are taken, we model the temporal dynamics of the energy level as a birth-death process. Using stopping/hitting time analysis, we quantify the uncertainty in the availability of each class of BSs due to finite battery capacity and inherent randomness in the energy harvesting process. Using this novel analytical approach, we prove that there is a fundamental limit on the availabilities, which cannot be surpassed by any uncoordinated strategy. We also construct an achievable scheme that achieves this upper limit on availability for each class of BSs.

\section{SySTEM MODEL}

We consider a $K$-tier cellular network consisting of $K$ different classes of BSs. For notational simplicity, define $\mathcal{K}=\{1,2, \ldots, K\}$. The locations of the BSs of the $k^{t h}$ tier are modeled by an independent PPP $\Phi_{k}$ of density $\lambda_{k}$. This model was first proposed for HCNs in [9] and has been 
validated since then both by empirical evidence [10] and theoretical arguments [11]. Each BS is assumed to have an energy harvesting module and an associated energy storage module, which is assumed to be its sole source of energy. The BSs across tiers may differ both in terms of how fast they harvest energy, i.e., the energy harvesting rate $\mu_{k}$ units per unit time, and how much energy they can store, i.e., the energy storage capacity $N_{k}$ units. While the absolute units of energy are irrelevant, we assume that the normalization is such that each user requires one unit of energy per unit time. This assumption can be easily relaxed to incorporate users requiring more than one unit of energy under sufficient randomization, but this case is not in the scope of the current work. We further assume that a $k^{t h}$ tier BS transmits to each user with a fixed power $P_{k}$. The target signal-to-interference-ratio (SIR) $\beta$ is the same for all the tiers.

The energy arrival process at a $k^{\text {th }}$ tier BS is modeled as a Poisson process with mean $\mu_{k}$. This assumption is based on the fact that most energy harvesting modules contain small sub-modules harvesting energy independently, e.g., small solar cells harvesting energy in a solar panel, where the net energy harvested can be argued to be a Binomial process, which approaches to the Poisson process in the limit when the number of sub-modules grow large. Interestingly, this model has been validated in literature using empirical measurements for a variety of real world energy harvesting modules, e.g., see [12]. Since the energy arrivals are random and the energy storage capacities are finite, there is some uncertainty associated with whether the BS has enough energy to serve users at a particular time or not. Under such a constraint, it is required that some of the BSs be kept OFF for the purpose of serving users and allowed to recharge while their load is handled by the neighboring BSs that are ON. Therefore, the BS can be in either of the two operational states: i) ON, or ii) OFF. The decision to toggle the operational state from one to another is taken by the operational strategies that can be broadly categorized into the following two classes.

Uncoordinated: In this class of strategies, the decision to toggle the state is taken by the BS independently of the operational states of the other BSs. For example, a BS may decide to toggle its operational state from ON to OFF if its current energy level reaches below a certain predefined level and toggle it back after harvesting enough energy. This class will be the main focus of this paper.

Coordinated: In this class of strategies, the decision to toggle the state of a particular BS is dependent upon the states of the other BSs. For example, the BSs may be partitioned into small clusters where only a few BSs in each cluster are turned ON. The decision may be taken by some central entity based upon the current load requirements.

For tractability, we focus on a time scale that is much longer than the scheduling block over which scheduling and cell selection decisions are taken. This allows us to consider the average effects of cell selection while determining the energy utilization rates for various classes of BSs as discussed in the next section. Further, due to uncertainty in the energy availability, all the BSs in the network may not always be available to serve users. This is made precise by defining

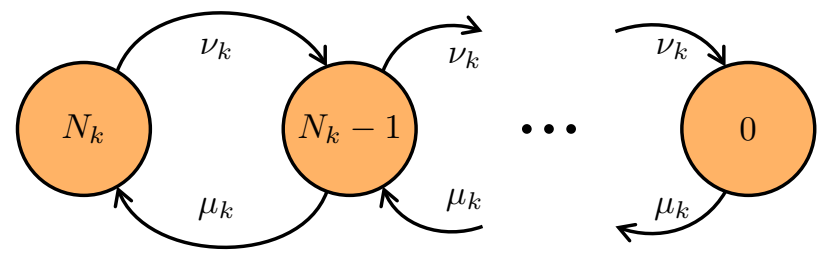

Fig. 1. Birth-death process modeling the temporal dynamics of the energy available at a $k^{t h}$ tier BS.

availability of a BS as follows.

Definition 1 (Availability). A BS is said to be available if it is in the ON state as a part of the operational policy and has enough energy to serve at least one user, i.e., has at least one unit of energy. The probability that a BS of tier $k$ is available is denoted by $\rho_{k}$, which may be different for different tiers of $B S s$ due to the differences in the capabilities of the energy harvesting modules and the load served.

For uncoordinated strategies, it is reasonable to assume that the current operational state of a BS is independent of all the other BSs, especially since the energy harvesting processes are assumed to be independent across the BSs. The coupling in the transmission of various BSs that arises due to interference and mobility is ignored. Under this independence assumption, the set of ON BSs of the $k^{\text {th }}$ tier form a PPP $\Phi_{k}^{\prime}$ with density $\lambda_{k} \rho_{k}$. This results from the fact that the independent thinning of a PPP leads to a PPP with appropriately scaled density [13]. As will be evident from the availability analysis in the next section, this abstraction is the key that makes this model tractable and leads to meaningful insights.

In the rest of this section, we introduce the propagation and cell selection models for which we restrict our attention to the BSs that are available. For notational ease, we define $\Phi^{\prime}=\cup_{k \in \mathcal{K}} \Phi_{k}^{\prime}$. We assume that a typical user is located at the origin, which is made possible by Slivnyak's theorem [9], [14]. The received power at the typical user from a $k^{\text {th }}$ tier BS located at $x_{k} \in \Phi_{k}^{\prime}$ is

$$
P\left(x_{k}\right)=P_{k} h_{x_{k}}\left\|x_{k}\right\|^{-\alpha},
$$

where $h_{x} \sim \exp (1)$ models Rayleigh fading, and $\left\|x_{k}\right\|^{-\alpha}$ represents standard power-law path loss with exponent $\alpha$, which is the same for all the tiers. For cell selection, we assume that each user connects to the BS that provides the highest long term power, i.e., small scale fading gain $h_{x}$ does not affect cell selection. Therefore, the typical user selects the BS located at $x$ if

$$
x=\arg \max _{y \in \Phi^{\prime}} P_{k}\|y\|^{-\alpha} .
$$

For this general system model, we now study the availabilities of different classes of BSs in the next section.

\section{AVAilability ANALYsis}

The main challenge in studying the model introduced in the previous section lies in characterizing how the energy available at the BS changes over time. Without loss of generality, we 


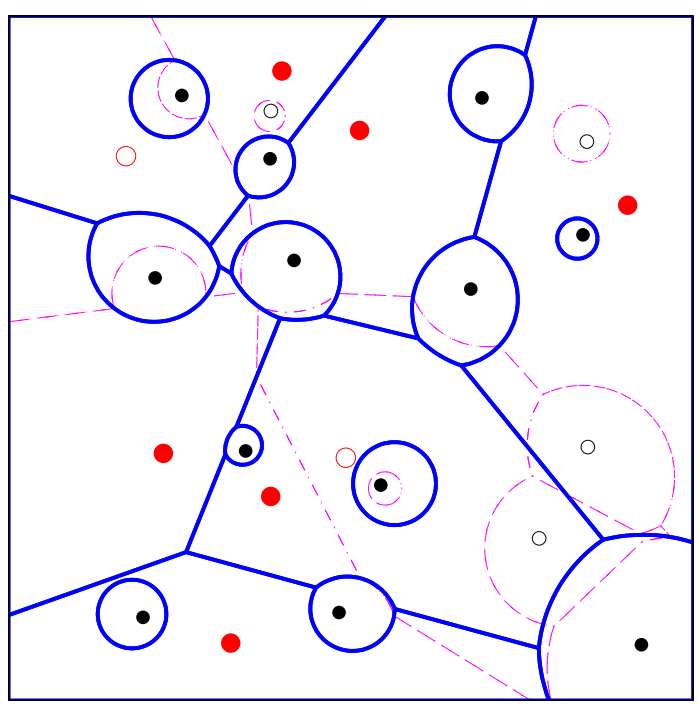

Fig. 2. Coverage regions for a two-tier cellular network. The unavailable BSs are denoted by hollow circles. The thin lines form coverage regions for the baseline case assuming all the BSs were available.

index the energy states of a $k^{t h}$ tier BS as $0,1, \ldots, N_{k}$, and model the temporal dynamics as a continuous time Markov chain (CTMC), in particular birth-death process, as shown in Fig. 1. When the BS is ON, the energy increases according to the energy harvesting rate and decreases at a rate that depends upon the number of users served by that BS. When the BS is OFF, it does not serve any users and hence the birth-death process reduces to a birth-only process. We now derive the rate $\nu_{k}$ at which the energy is utilized in closed form below.

\section{A. Modeling Energy Utilization Rate}

Before modeling the energy utilization rate, there are two noteworthy points. First, if a BS is not available, the load originating from its original area of coverage is directed to the nearby BSs that are available, thus increasing their effective load. Equivalently, the coverage areas of the BSs that are available get expanded to cover for the BSs that are not available, as shown in Fig. 2. The second one is given in the following remark.

Remark 1 (Control channel coverage). The control channel coverage, i.e., the probability that the received SIR is greater than the target, is independent of the densities of the BSs in an interference limited network when the target SIR is the same for all tiers [9], [15]. While this result will be familiar to those exposed to recent coverage probability analysis using stochastic geometry [9], [15], this is not required for the current paper, except the interpretation that the density of users effectively served by the network is independent of the effective densities of the BSs and hence independent of $\left\{\rho_{k}\right\}$.

Assuming fixed energy expenditure for control signaling, only the users that are in control channel coverage will result in additional energy expenditure at the BS. We denote the density of such users by $\lambda_{u}$. Each user is assumed to require one unit of energy per unit time such that the net energy utilization process at each BS can be modeled as a Poisson process with mean defined by the average number of users it serves. It should be noted that the assumption of unit energy usage is without any loss of generality and is made to simplify the notation. To find the average number of users served by a BS of each class, we first need to define its service area which will, in general, be different for different tiers due to the differences in the transmit powers as evident from Fig. 2. The service area can be formally defined as follows.

Definition 2 (Service area). The service area $\mathcal{A}_{k}\left(x_{k}\right) \subset \mathbb{R}^{2}$ of the $k^{\text {th }}$-tier BS located at $x_{k} \in \Phi_{k}^{\prime}$ is defined as

$$
\mathcal{A}_{k}\left(x_{k}\right)=\left\{z \in \mathbb{R}^{2}: x_{k}=\arg \max _{j \in \mathcal{K}, x_{j} \in \Phi_{j}^{\prime}} P_{j}\left\|x_{j}-z\right\|^{-\alpha}\right\} .
$$

To find the average service area of a $k^{t h}$ tier BS, we first derive the probability that a typical user connects with a BS of that tier. We denote this by $\mathcal{P}_{k}$ which is given next.

Lemma 1 (Selection probabilities). For the cell selection model introduced in the last section, the probability that a typical user connects to a BS of $k^{\text {th }}$ tier is given by

$$
\mathcal{P}_{k}=\frac{\lambda_{k} \rho_{k} P_{k}^{\frac{2}{\alpha}}}{\sum_{j=1}^{K} \lambda_{j} \rho_{j} P_{j}^{\frac{2}{\alpha}}}
$$

The proof follows from Lemma 1 of [15] using the fact that the density of available BSs of $k^{t h}$ tier is $\rho_{k} \lambda_{k}$. The equivalence of the selection probabilities and the average area served by a particular tier is given below.

Remark 2 (Average area). Due to the ergodicity of PPP, the selection probability given by Lemma 1 is equal to the average fraction of area covered by the corresponding tier.

This leads to the following corollary of Lemma 1 about the average service area of a BS of the $k^{\text {th }}$ tier.

Corollary 1 (Average service area). The average service area of a $k^{\text {th }}$ tier $B S$ is given by

$$
\mathbb{E}\left[\mathcal{A}_{k}\right]=\frac{P_{k}^{\frac{2}{\alpha}}}{\sum_{j=1}^{K} \lambda_{j} \rho_{j} P_{j}^{\frac{2}{\alpha}}} .
$$

The proof follows from the fact that $\mathbb{E}\left[\mathcal{A}_{k}\right]=\mathcal{P}_{k} / \lambda_{k} \rho_{k}$, where $\lambda_{k} \rho_{k}$ is the effective density of the $k^{t h}$ tier BSs. Using this result, the average number of users served by a BS of $k^{t h}$ tier, equivalently the energy utilization rate, is now given by the next corollary.

Corollary 2 (Energy utilization rate). The energy utilization rate, i.e., the number of units of energy required per second, at a BS of $k^{\text {th }}$ tier is given by

$$
\nu_{k}=\lambda_{u} \mathbb{E}\left[\mathcal{A}_{k}\right]=\frac{\lambda_{u} P_{k}^{\frac{2}{\alpha}}}{\sum_{j=1}^{K} \lambda_{j} \rho_{j} P_{j}^{\frac{2}{\alpha}}} .
$$

It should be noted that the availabilities of various tiers are still unknown and even if all the system parameters are given, it is still not possible to determine the energy utilization rate from the above expression. This will lead to fixed point expressions in terms of availabilities as discussed in detail 
later in this section. It is also worth mentioning that the energy utilization rate derived above is just for the service of the active users. There are some other components of energy usage, e.g., control channel signaling and backhaul that are not modeled. While we can incorporate their effect in the current model by assuming fixed energy expenditure and deducing it directly from the energy arrival rate, a more formal treatment of these components is left for future work.

\section{B. Availabilities for a Simple Operational Strategy}

After deriving the energy utilization rate in Corollary 2 and recalling that the energy harvesting rate is $\mu_{k}$, we can, in principle, derive BS availabilities for a variety of uncoordinated operational strategies. To fix the key ideas, we begin by looking at a very simple strategy in which a BS is said to be available when it is not in energy state 0 , i.e., it has at least one unit of energy. The availability of a $k^{t h}$ tier BS under this strategy can be derived directly from the stationary distribution of the birth-death process as follows:

$$
\begin{aligned}
\rho_{k} & =1-\left(\frac{1-\frac{\mu_{k}}{\nu_{k}}}{1-\left(\frac{\mu_{k}}{\nu_{k}}\right)^{N_{k}+1}}\right) \\
& =1-\left(\frac{1-\frac{\mu_{k} \sum_{j=1}^{K} \lambda_{j} \rho_{j} P_{j}^{\frac{2}{\alpha}}}{\lambda_{u} P_{k}^{\frac{2}{\alpha}}}}{1-\left(\frac{\mu_{k} \sum_{j=1}^{K} \lambda_{j} \rho_{j} P_{j}^{\frac{2}{\alpha}}}{\lambda_{u} P_{k}^{\frac{2}{\alpha}}}\right)^{N_{k}+1}}\right) .
\end{aligned}
$$

Interestingly we get a set of $K$ fixed point equations in terms of $\left\{\rho_{k}\right\}$, one for each tier. Due to the form of these equations, it is not possible to derive closed form expressions for $\left\{\rho_{k}\right\}$. However, it is possible to comment on the necessary and sufficient condition for the existence of a positive solution.

Theorem 1. Necessary and sufficient condition for the existence of a positive solution $\rho_{k}>0, \forall k \in \mathcal{K}$ for the system of fixed point equations given by (7) is

$$
\sum_{k=1}^{K} \lambda_{k} \mu_{k}>\lambda_{u}
$$

Proof: For $\rho_{k} \neq 0$, the fixed point equation (7) can be expressed as

$$
\rho_{k}=1-\left(\frac{1-\kappa_{k} \rho_{k}}{1-\left(\kappa_{k} \rho_{k}\right)^{N_{k}+1}}\right),
$$

where

$$
\kappa_{k}=\frac{\mu_{k} \sum_{j=1}^{K} \lambda_{j} \rho_{j} P_{j}^{\frac{2}{\alpha}}}{\lambda_{u} \rho_{k} P_{k}^{\frac{2}{\alpha}}} .
$$

We first show the fixed point equation (9) has a positive solution $\rho_{k} \leq 1$ iff $\kappa_{k}>1$. For notational simplicity, denote the RHS of (9) by $g\left(\rho_{k}\right)$ assuming other parameters as constants. It is easy to note that $g\left(\rho_{k}\right)$ is an increasing function of $\rho_{k}$ and that $g\left(\rho_{k}\right)<1$ for finite $N_{k}$. Therefore, for existence, it suffices to show that $g^{\prime}\left(\rho_{k}\right)>1$ for $\rho_{k} \rightarrow 0$. Furthermore, it is easy to show that $g^{\prime}\left(\rho_{k}\right)=\kappa_{k}$ for $\rho_{k} \rightarrow 0$, which leads to the condition $\kappa_{k}>1$ for the existence of the solution. This leads to the following set of inequalities for $1 \leq k \leq K$

$$
\frac{\mu_{k} \sum_{j=1}^{K} \lambda_{j} \rho_{j} P_{j}^{\frac{2}{\alpha}}}{\lambda_{u} \rho_{k} P_{k}^{\frac{2}{\alpha}}}>1 .
$$

It is easy to show that $(8) \Leftrightarrow(11)$, which completes the proof sketch. Further details are delegated to [16] due to the shortage of space.

Note that while the uniqueness of the fixed point is not required for this paper, it is possible to show that under (8), the fixed point solution is also unique [16]. Nevertheless, condition (8) is basically an energy conservation law, i.e., the net energy harvested by all the tiers should be greater than the effective energy required by all the users. A slightly more interesting interpretation is in terms of tradeoffs between various over-provisioning options, such as BS density and energy storage capacity, as discussed later in this section.

Remark 3 (Effect of battery capacity on availability). Note that the function $g\left(\rho_{k}\right)$ is an increasing function of $N_{k}$ from which it directly follows that the availability of a particular class of BSs increases with the increase in the battery capacity.

Remark 4 (Effect of availabilities of other tiers on $\rho_{k}$ ). It is easy to see that $g\left(\rho_{k}\right)$ is an increasing function of $\rho_{j}, j \neq k$. This implies that the availability of $k^{\text {th }}$ tier increases if the availability of one or more of the other tiers is increased. This is consistent with the intuition that if the availability of any tier is increased, the effective load on other tiers decreases hence increasing their availabilities.

We now develop tools to study availabilities for a general energy-based uncoordinated strategy using stopping/hitting time analysis. We show that the simple strategy discussed above maximizes the BS availabilities over the space of uncoordinated strategies, where each BS's decision to toggle its operational state is solely dependent upon its current energy level.

\section{Availabilities for any General Uncoordinated Strategy}

We focus on a general set of strategies $\left\{\mathcal{S}\left(N_{k \text { min }}, N_{k c}\right)\right\}$ in which a BS toggles its state based solely on its current energy level, i.e., a $k^{\text {th }}$ tier BS toggles to OFF state when its energy level reaches some level $N_{k \text { min }}$ and toggles back to ON state when the energy level reaches some predefined cutoff value $N_{k c}>N_{k \text { min }}$ as shown in Fig. 3. First note that for the proposed model it is strictly suboptimal to toggle to OFF state at any $N_{\min } \neq 0$, since it effectively reduces the storage capacity to $N_{k}-N_{k \text { min. }}$. Therefore, without any loss of generality we fix $N_{k \text { min }}=0$ (for all tiers) and denote this strategy by $\mathcal{S}_{k}\left(N_{k c}\right)$. For this strategy, we denote the time for which a $k^{t h}$ tier BS is in the ON state after it toggles from the OFF state by $J_{k_{1}}\left(N_{k c}\right)$ and the time for which it remains in the OFF state after toggling from the ON state by $J_{k_{2}}\left(N_{k c}\right)$. The cutoff value in the arguments will be dropped for notational simplicity wherever appropriate. The cycles of ON and OFF times go on as shown in Fig. 3. It is worth highlighting that 


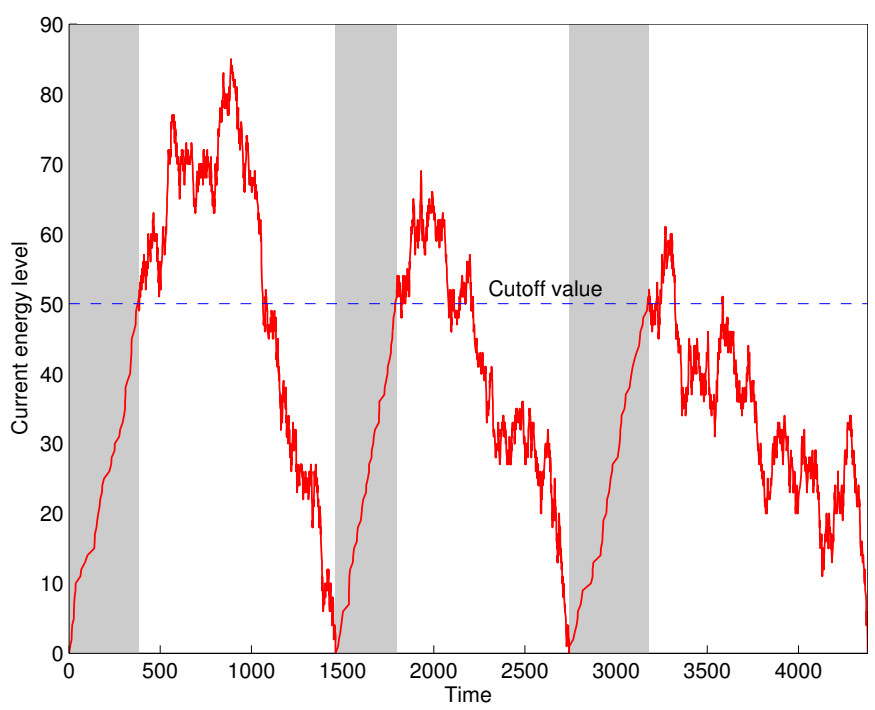

Fig. 3. Illustration of how the energy level changes over time. The time for which BS is in OFF state is shaded. The unit of time is irrelevant.

both $J_{k_{1}}$ and $J_{k_{2}}$ are in general random variables due to the randomness involved in both the energy availability and its utilization, e.g., $J_{k_{1}}$ can be formally expressed as

$$
J_{k_{1}}\left(N_{k c}\right)=\inf \left\{t: \mathcal{E}_{k}(t)=0 \mid \mathcal{E}_{k}(0)=N_{k c}\right\}
$$

where $\mathcal{E}_{k}(t)$ denotes the current energy level of a $k^{t h}$ tier BS at time $t$. For this setup, the availabilities depend only on the means of $J_{k_{1}}$ and $J_{k_{2}}$ as shown in the following Lemma.

Lemma 2 (Availability). The availability of a $k^{\text {th }}$ tier BS for any operational strategy can be expressed as

$$
\rho_{k}=\frac{\mathbb{E}\left[J_{k_{1}}\right]}{\mathbb{E}\left[J_{k_{1}}\right]+\mathbb{E}\left[J_{k_{2}}\right]}=\frac{1}{1+\frac{\mathbb{E}\left[J_{k_{2}}\right]}{\mathbb{E}\left[J_{k_{1}}\right]}},
$$

where $\mathbb{E}\left[J_{k_{1}}\right]$ is the mean time a BS spends in the ON state and $\mathbb{E}\left[J_{k_{2}}\right]$ is the mean time it spends in the OFF state.

Proof: For a particular realization, let $\left\{J_{k_{1}}^{(i)}\right\}$ and $\left\{J_{k_{2}}^{(i)}\right\}$ be the sequences of $\mathrm{ON}$ and OFF times, respectively, with $i$ being the index of the ON-OFF cycle. The availability can now be expressed as the fraction of time a BS spends in the ON state, which leads to

$$
\rho_{k}=\lim _{n \rightarrow \infty} \frac{\sum_{i=1}^{n} J_{k_{1}}^{(i)}}{\sum_{i=1}^{n} J_{k_{1}}^{(i)}+\sum_{i=1}^{n} J_{k_{2}}^{(i)}} .
$$

The proof follows by dividing both the numerator and the denominator by $n$ and invoking the law of large numbers.

To set up a fixed point equation similar to (7) for the strategy $\mathcal{S}_{k}\left(N_{k c}\right)$, we need closed form expressions for the mean ON time $\mathbb{E}\left[J_{k_{1}}\right]$ and the mean OFF time $\mathbb{E}\left[J_{k_{2}}\right]$. Note that the OFF time for $\mathcal{S}_{k}\left(N_{k c}\right)$ is simply the time required to harvest $N_{k c}$ units of energy, which is the sum of $N_{k c}$ exponentially distributed random variables, each with mean $1 / \mu_{k}$. Therefore, $\mathbb{E}\left[J_{k_{2}}\right]=\frac{N_{k c}}{\mu_{k}}$, which simplifies the availability expression to

$$
\rho_{k}=\frac{1}{1+\frac{N_{k c}}{\mu_{k} \mathbb{E}\left[J_{k_{1}}\right]}} .
$$

To derive $\mathbb{E}\left[J_{k_{1}}\right]$, we first define the generator matrix for the birth-death process corresponding to a $k^{\text {th }}$ tier BS as $A_{k}=$

$$
\left[\begin{array}{cccccc}
-\mu_{k} & \mu_{k} & 0 & \cdots & 0 & 0 \\
\nu_{k} & -\mu_{k}-\nu_{k} & \mu_{k} & \cdots & 0 & 0 \\
0 & \nu_{k} & -\mu_{k}-\nu_{k} & \cdots & 0 & 0 \\
\vdots & \vdots & & \ddots & & \\
0 & 0 & 0 & \cdots & \nu_{k} & -\nu_{k}
\end{array}\right] \text {, }
$$

where the states are ordered in the ascending order of the energy levels, i.e., the first column corresponds to the energy level 0 . To complete the derivation, we need the following technical result. Please refer to Proposition 5.7.2 of [17] for a more general version of this result and its proof.

Lemma 3 (Mean hitting time). If the embedded discrete Markov chain of the CTMC is irreducible then the mean time to hit energy level 0 (state 1) starting from energy level $i$ (state $i+1)$ is

$$
\mathbb{E}\left[J_{k_{1}}(i)\right]=\left(\left(-B_{k}\right)^{-1} \mathbb{1}\right)(i),
$$

where $\mathbb{1}$ is a column vector of all $1 s$ and $B_{k}$ is a $\left(N_{k}-1\right) \times$ $\left(N_{k}-1\right)$ sub-matrix of $A_{k}$ obtained by deleting first row and column of $A_{k}$.

For $A_{k}$ given by (16), we can derive a closed form expression for each element of $\left(-B_{k}\right)^{-1}$ after some algebraic manipulations. The $(i, j)^{t h}$ element can be expressed as

$$
\left(-B_{k}\right)^{-1}(i, j)=\frac{1}{\nu_{k}^{j}} \sum_{n=1}^{\min (i, j)} \mu_{k}^{j-n} \nu_{k}^{n-1} .
$$

Now substituting (18) back in (17) gives us the mean ON time for any strategy $\mathcal{S}_{k}\left(N_{k c}\right)$, which when substituted in (15) gives a fixed point equation in $\left\{\rho_{k}\right\}$ similar to (7), as illustrated below for the two policies of interest.

1) Policy $1\left(\mathcal{S}_{k}(1)\right)$ : In this policy, each BS serves users until it depletes all its energy after which it toggles to OFF state. It toggles back to $\mathrm{ON}$ state after it has harvested one unit of energy. Using (17) and (18), the mean ON time $\mathbb{E}\left[J_{k 1}\right]$ for this policy can be expressed as

$$
\mathbb{E}\left[J_{k_{1}}\right]=\frac{1}{\nu_{k}} \frac{1-\left(\frac{\mu_{k}}{\nu_{k}}\right)^{N_{k}}}{1-\left(\frac{\mu_{k}}{\nu_{k}}\right)},
$$

which when substituted into (15) leads to the same fixed point equation as (7), establishing the equivalence between this policy and the one studied in the previous subsection.

2) Policy $2\left(\mathcal{S}_{k}\left(N_{k}\right)\right)$ : As in the above policy, each BS serves users until it depletes all its energy after which it toggles to OFF state. Under this policy, the BS waits in the OFF state until it harvests $N_{k}$ units of energy, i.e., it is completely charged. Using (17) and (18), $\mathbb{E}\left[J_{k_{1}}\right]$ can be computed as

$$
\mathbb{E}\left[J_{k_{1}}\right]=\frac{1}{\mu_{k}-\nu_{k}} \frac{\mu_{k}}{\nu_{k}} \frac{1-\left(\frac{\mu_{k}}{\nu_{k}}\right)^{N_{k}}}{1-\left(\frac{\mu_{k}}{\nu_{k}}\right)}-\frac{N_{k}}{\mu_{k}-\nu_{k}},
$$

which when substituted in (15) gives the fixed point equation for this policy. 


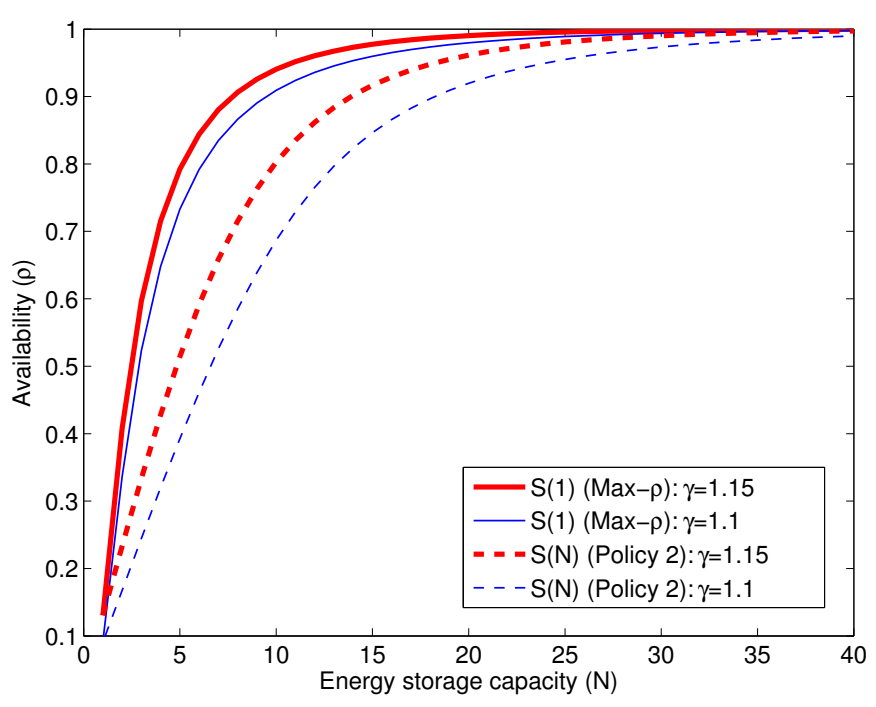

Fig. 4. Availabilities for strategies $\mathcal{S}(1)$ and $\mathcal{S}(N)$ for values of the overprovisioning factor $\gamma$.

Before providing more details for the above policies, we prove the following theorem, which establishes a fundamental upper limit on the availabilities of various types of BSs that cannot be surpassed by any uncoordinated strategy.

Theorem 2. For a given $K$-tier $H C N$, the availabilities of all the classes of BSs are jointly maximized if each tier follows strategy $\mathcal{S}_{k}(1)$. The availabilities are strictly lower if any one or more tiers follow $\mathcal{S}_{k}(i)$ with $i>1$.

Proof: From (15), note that the availability for a $k^{\text {th }}$ tier BS is maximized if $\mathbb{E}\left[J_{k_{1}}\left(N_{k c}\right)\right] / N_{k c}$ is maximized. Using (17) and (18), it is straightforward to show that

$$
\arg \max _{1 \leq i \leq N_{k}} \frac{\mathbb{E}\left[J_{k_{1}}(i)\right]}{i}=1 \text {. }
$$

The proof now follows from the fact that if any tier follows strategy $\mathcal{S}_{k}(i)(i>1)$, its availability will be strictly lower than that of $\mathcal{S}_{k}(1)$, which increases the effective load on other tiers and hence decreases their availabilities, as discussed in Remark 4 . Therefore, to jointly maximize the availabilities of all the tiers, each tier has to follow $\mathcal{S}_{k}(1)$.

\section{Discussion on Tradeoffs in Over-Provisioning}

For conciseness, we limit this concluding discussion to a single tier network and define the over-provisioning factor $\gamma=\lambda \mu / \lambda_{u}$ as the ratio of the net energy harvested per unit area per unit time and the net energy utilized per unit area per unit time. For various values of $\gamma$, we numerically solve the fixed point equation for policies $\mathcal{S}(1)$ and $\mathcal{S}(N)$ and plot the results in Fig. 4. We note that there is a tradeoff between the battery capacity and over-provisioning factor, i.e., to achieve the same availability, either we can have a higher over-provisioning in terms of BS density or have a higher energy storage capacity. More interestingly, we observe that for reasonable energy storage capacities, the availability probability approaches unity, which shows that this setup, despite randomness in the energy harvesting and utilization, is surprisingly reliable if designed properly. We also observe that $\mathcal{S}(1)$ outperforms $\mathcal{S}(N)$ as expected by Theorem 2 .

\section{CONCLUSIONS}

In this paper, we have developed a comprehensive framework to study self-powered HCNs. Using novel tools from stochastic geometry and random walk theory, we quantified the uncertainty in BS availability due to the finite battery capacity and inherent randomness in energy harvesting. We further derived a fundamental limit on the availability of each class of BSs, which cannot be surpassed by any uncoordinated strategy. This work has many extensions, such as incorporating more accurate energy expenditure model taking into account energy spent on backhaul and control signaling. Another possible extension is to incorporate the BS availability results in short time scale analysis while studying key performance metrics such as downlink coverage and rate.

\section{REFERENCES}

[1] J. G. Andrews, "Seven ways that HetNets are a cellular paradigm shift," IEEE Communications Magazine, vol. 51, no. 3, pp. 136 - 144, Mar. 2013.

[2] H. S. Dhillon, R. K. Ganti, and J. G. Andrews, "Load-aware modeling and analysis of heterogeneous cellular networks," IEEE Trans. on Wireless Communications, vol. 12, no. 4, pp. 1666 - 1677, Apr. 2013.

[3] S. Hur, T. Kim, D. J. Love, J. V. Krogmeier, T. A. Thomas, and A. Ghosh, "Multilevel millimeter wave beamforming for wireless backhaul," in Proc., IEEE Globecom Workshops, Houston, TX, 2011.

[4] S. Sudevalayam and P. Kulkarni, "Energy harvesting sensor nodes: Survey and implications," IEEE Communications Surveys and Tutorials, vol. 13, no. 3, pp. 443 - 461, Third Quarter 2011.

[5] K. Huang, "Throughput of wireless networks powered by energy harvesting," in Proc., IEEE Asilomar, Monterey, CA, Nov. 2011.

[6] J. Yang and S. Ulukus, "Optimal packet scheduling in an energy harvesting communication systems," IEEE Trans. on Communications, vol. 60, no. 1, pp. 220 - 230, Jan. 2012.

[7] K. Tutuncuoglu and A. Yener, "Optimum transmission policies for battery limited energy harvesting nodes," IEEE Trans. on Wireless Communications, vol. 11, no. 3, pp. 1180 - 1189, Mar. 2012.

[8] O. Ozel, K. Tutuncuoglu, J. Yang, S. Ulukus, and A. Yener, "Transmission with energy harvesting nodes in fading wireless channels: Optimal policies," IEEE Journal on Sel. Areas in Communications, vol. 29, no. 8, pp. $1732-1743$, Sep. 2011.

[9] H. S. Dhillon, R. K. Ganti, F. Baccelli, and J. G. Andrews, "Modeling and analysis of K-tier downlink heterogeneous cellular networks," IEEE Journal on Sel. Areas in Communications, vol. 30, no. 3, pp. 550 - 560, Apr. 2012.

[10] D. B. Taylor, H. S. Dhillon, T. D. Novlan, and J. G. Andrews, "Pairwise interaction processes for modeling cellular network topology," in Proc., IEEE Globecom, Anaheim, CA, Dec. 2012.

[11] B. Blaszczyszyn, M. K. Karray, and H.-P. Keeler, "Using Poisson processes to model lattice cellular networks," available online: arxiv.org/abs/1207.7208.

[12] S. Roundy, P. K. Wright, and J. M. Rabaey, Energy Scavenging for Wireless Sensor Networks: With Special Focus on Vibrations. Norwell, MA: Kluwer Academic Publishers, 2004.

[13] J. F. C. Kingman, Poisson Processes. Oxford University Press, 1993.

[14] D. Stoyan, W. S. Kendall, and J. Mecke, Stochastic Geometry and Its Applications, 2nd ed. Chichester: John Wiley and Sons, 1995.

[15] H.-S. Jo, Y. J. Sang, P. Xia, and J. G. Andrews, "Heterogeneous cellular networks with flexible cell association: A comprehensive downlink SINR analysis," IEEE Trans. on Wireless Communications, vol. 11, no. 10, pp. 3484 - 3495, Oct. 2012.

[16] H. S. Dhillon, Y. Li, P. Nuggehalli, Z. Pi, and J. G. Andrews, "Fundamentals of heterogeneous cellular networks with energy harvesting," submitted to IEEE Tran. Wireless Communications, Jul. 2013. Available online: arxiv.org/abs/1307.1524.

[17] S. I. Resnick, Adventures in Stochastic Processes. Boston: Birkhäuser, 2005. 